\title{
Continuous Network Design Based on the Paired Combinatorial Logit Sto- chastic User Equilibrium Model
}

\author{
Xuefei $\mathrm{Li}^{1,2, *}$ and Maoxiang Lang ${ }^{1}$ \\ ${ }^{I}$ MOE Key Laboratory for Urban Transportation Complex Systems Theory and Technology, Beijing Jiaotong Universi- \\ ty, Beijing, 100044, China \\ ${ }^{2}$ National Railway Track Test Center, China Academy of Railway Sciences, Beijing, 100015, China
}

\begin{abstract}
In order to design the traffic network more accurately, the bi-level programming model for the continuous network design problem based on the paired combinatorial Logit stochastic user equilibrium model is proposed in this study. In the model, the paired combinatorial Logit stochastic user equilibrium model which is used to characterize the route choice behaviors of the users is adopted in the lower level model, and the minimum summation of the system total costs and investment amounts is used in the upper objective function. The route-based self-regulated averaging (SRA) algorithm is designed to solve the stochastic user equilibrium model and the genetic algorithm (GA) is designed to get the optimal solution of the upper objective function. The effectiveness of the proposed combining algorithm which contains GA and SRA is verified by using a simple numerical example. The solutions of the bi-level models which use the paired combinatorial Logit stochastic user equilibrium model in the lower level model with different demand levels are compared. Finally, the impact of the dispersion coefficient parameter which influences the decision results of the network design problem is analyzed.
\end{abstract}

Keywords: Genetic algorithm, network design, paired combinatorial logit, SRA.

\section{INTRODUCTION}

Network Design Problem (NDP) is one of the hot issues in the field of traffic studies, and the research of this problem has high theoretical and practical significances. Traffic network design problem is within a certain range of investment constraints, and under the consideration of the route choice behaviors of users, by expansion the capacity of certain links or adding new links in the traffic network, so as to achieve the purpose of getting the optimal system indicator [1]. In the traffic network, to improve link capacities is called continuous network design problem (CNDP), and to add new links is called discrete network design problem (DNDP).

The research content of this study is a continuous network design problem. For CNDP, many experts and scholars have done a lot of researches. Song and Gao (1998) [2] made the difference quotient in which the increasing amount of balanced flow towards the link capacity was the approximation of the differential quotient, and designed the BLABD algorithm to solve the continuous equilibrium network design problem. Yang et al. (2009) [3] adopted the simulated annealing algorithm to solve the urban traffic continuous network design problem and made the sensitivity analysis of different parameters. Xu and Gao (2006) [4] established a bilevel programming model of urban traffic network design which considered the reliability of ability, and designed its chaos optimization heuristics algorithm. Wang and Lo (2010) [5] transformed the flow balancing constraint into a series of mixed-integer constraints and made the travel time function linearization, this CNDP could be solved by the existing mixed integer programming methods and got the optimal solution. Chiou (2005) [6] designed a descent method based on gradient algorithm for solving the continuous traffic network design problem, and designed another four improved algorithms. Wang et al. (2013) [7] constructed a continuous traffic network design model which considered the multi-user class and designed a corresponding cut constraint algorithm.

In the researches of traffic network design, the bi-level programming models are used generally. The objective functions in the upper level are normally the system travel costs minimum, network spare capacity maximum, consumer surplus maximum and multi-objective optimization etc. [8]. The objective function in the lower level needs to consider the users' route choice behaviors in urban traffic networks in order to avoid the condition that the traffic situation worse and system total costs increase which are caused by Braess paradox [9]. The objective function in the lower level normally adopts the deterministic or stochastic user equilibrium models. In the existing traffic network design models, the stochastic user equilibrium models (SUE) used in the lower level function are mainly traditional Logit-based models. However, due to the independence of irrelevant alternatives (IIA) and other undesirable characteristics which exist in the Logit model [10], it could not reflect the true influences on the route choice behaviors of users which are caused by the 
path overlapping problem. Therefore, it is necessary to adopt the stochastic user equilibrium model which could overcome the overlapping problem in the lower level model of the CNDP model, so as to reflect the route choice behaviors of the users towards the improved link capacity more objectively.

With the development of Generalized Extreme Value (GEV) [11] models, the paired combinatorial Logit (PCL) and other discrete choice models which could overcome the IIA property of traditional Logit model have been proposed, and the corresponding stochastic user equilibrium model based on the paired combinatorial Logit (PCL-SUE) [12] is also established. In this study, the PCL-SUE model is used as the lower level model of CNDP and a new traffic network design model is built, and the genetic algorithm is designed to solve the new bi-level programming model of the continuous traffic network design. Finally, by a numerical example, the impacts on the calculation results of CNDP model established in this study under different demand levels and dispersion coefficient parameters are analyzed.

\section{PCL-SUE MODEL}

In the paired combinatorial Logit (PCL) model, the probability of choosing route $k$ between OD pair $r s$ is as following [12]:

$$
P^{r s}(k)=\frac{\sum_{j \neq k} e^{\frac{-\theta c_{k}^{r s}}{1-\sigma_{k j}^{s s}}}\left(1-\sigma_{k j}^{r s}\right)\left(e^{\frac{-\theta c_{k s}^{r s}}{1-\sigma_{l j}^{r s}}}+e^{\frac{-\theta c_{j}^{r s}}{1-\sigma_{k j}^{r s s}}}\right)^{-\sigma_{k j}^{r s}}}{\sum_{l=1}^{n-1} \sum_{h=l+1}^{n}\left(1-\sigma_{l h}^{r s}\right)\left(e^{\frac{-\theta c_{c}^{s s}}{1-\sigma_{l h}^{s s}}}+e^{\frac{-\theta c_{l}^{r s}}{1-\sigma_{l h}^{s s}}}\right)^{1-\sigma_{l h}^{r s}}}
$$

where $c_{k}^{r s}, c_{j}^{r s}, c_{l}^{r s}$ and $c_{h}^{r s}$ are the generalized costs of route $k, j, l$ and $h$ between OD pair $r s$, respectively. $\theta$ is dispersion coefficient. $\sigma_{k j}^{r s}$ and $\sigma_{l h}^{r s}$ are the indexes of similarity between route $k$ and $j$, and between route $l$ and $h$ in OD pair $r s$, respectively.

Based on the choice probability of paired combinatorial Logit model, the PCL-SUE equivalent mathematical programming model could be established as following [12]:

$$
\begin{aligned}
& \min Z=\sum_{a} \int_{0}^{x_{a}} t_{a}(w) d w+\frac{1}{\theta} \sum_{r s} \sum_{k} \sum_{j \neq k}\left(1-\sigma_{k j}^{r s}\right) f_{k(k j)}^{r s} \ln \left(\frac{f_{k(k j)}^{r s}}{1-\sigma_{k j}^{r s}}\right) \\
& \quad+\frac{1}{\theta} \sum_{r s} \sum_{k=1}^{n-1} \sum_{j=k+1}^{n} \sigma_{k j}^{r s}\left(f_{k(k j)}^{r s}+f_{j(k j)}^{r s}\right) \ln \left(\frac{f_{k(k j)}^{r s}+f_{j(k j)}^{r s}}{1-\sigma_{k j}^{r s}}\right) \\
& \text { s.t. } \sum_{k} \sum_{j \neq k} f_{k(k j)}^{r s}=q_{r s} \\
& x_{a}=\sum_{r s} \sum_{k} \sum_{j \neq k} f_{k(k j)}^{r s} \delta_{a, k}^{r s} \quad \forall a \\
& f_{k(k j)}^{r s} \geq 0 \quad \forall k, j, r s
\end{aligned}
$$

where $q_{r s}$ is the travel demand between OD pair $r s$, it is set fixed value. $f_{k(k j)}^{r s}$ is the flow on route $k$ (of the route pair $k j$ ) between OD pair $r s$, and $x_{a}$ is the flow on link $a$. Eq.(2) is the objective function to get the minimum value, Eq.(3) is the relationship between the route flow and OD travel demand, Eq.(4) is the relationship between link flow and route flow, Eq.(5) is the nonnegative constraints of the route flow. The derived and proved process of PCL-SUE model could be found in paper [12].

\section{CNDP MODEL BASED ON PCL-SUE}

The PCL-SUE model is applied in the CNDP model in this study. The CNDP model is a bi-level programming model, the minimum summation of the system total costs and investment amounts is adopted as the upper level objective function and the PCL-SUE model is used in the lower level. The upper level model used in this study is as follows [8]:

$$
\begin{aligned}
& \min \quad D=\sum_{a} t_{a}\left(x_{a}(\mathbf{y}), y_{a}\right) x_{a}(\mathbf{y})+\psi \sum_{a} G_{a}\left(y_{a}\right) \\
& \text { s.t. } y_{a} \geq 0, \quad \forall a
\end{aligned}
$$

where $t_{a}\left(x_{a}(\mathbf{y}), y_{a}\right)$ is the travel cost on link $a, x_{a}(\mathbf{y})$ is the flow on link $a, G_{a}\left(y_{a}\right)$ is the investment function of capacity expansion on link $a, \psi$ is the coefficient to match the units of investment costs and system total travel costs, and $y_{a}$ is the value of capacity expansion on link $a$.

The PCL-SUE model is the lower level model. In the bilevel programming model of CNDP, the travel cost function of the links $t_{a}\left(x_{a}\right)$ needs to be transformed to $t_{a}\left(x_{a}(\mathbf{y}), y_{a}\right)$ which contain the improved value of the link capacity, the equilibrium link flow $x_{a}$ also needs to be transformed to $x_{a}(\mathbf{y})$ which contain the expansion value of the link capacity, and the other parts are the same as PCL-SUE model. After the expansion capacity values of the links $\mathbf{y}=\left\{y_{a}\right\}$ are determined in the upper level model, it is left to solve the PCL-SUE model in the lower level model.

\section{ALGORITHM DESIGN}

The solving process of bi-level programming model is relatively complex in general, which is because the bi-level programming is a NP-Hard problem, and there is no corresponding polynomial algorithm, and because bi-level programming problems are generally non-convex, therefore it is difficult to find their global optimal solutions. Genetic algorithm (GA) [13] has a strong global search capability, and is a robust search algorithm for optimizing calculation of complex system, therefore the genetic algorithm is adopted to solve the proposed bi-level programming model in this study. In the proposed algorithm, the genetic algorithm is 
used to solve the upper level model and the lower level model is seen as the constraints of the upper level model, and plays the role of variable transfer in the bi-level programming model. There are several kinds of methods to solve the PCL-SUE model in the lower level, and we use the selfregulated averaging (SRA) algorithm proposed by Liu et al [14] to solve the PCL-SUE model.

(1) Algorithm of the lower level model

After the capacity expansion values of links $\mathbf{y}=\left\{y_{a}\right\}$ are determined, the lower level model is transferred to a typical PCL-SUE problem. The calculation steps of route-based self-regulated averaging algorithm are as following:

Step1: Determination of the valid route set $K^{r s}$ between every OD pair.

Step 2: Initialization. According to the free flow travel cost $t_{a}^{(0)}=t_{a}(0)$, calculate the travel cost $c_{k}^{r s}$ of all valid routes, and then get the initial route flow $f_{k}^{r s(d)}$ according to Eq.(1) and $f_{k}^{r s}=q_{r s} \cdot P^{r s}(k)$, let iteration number $d=1$.

Step3: Update. Get the link flow $x_{a}^{(d)}$ according to $f_{k}^{r s(d)}$ and Eq.(4), let $t_{a}^{(d)}=t_{a}\left(x_{a}^{(d)}\right)$.

Step 4: Determination of the descent direction. According to the current travel time of link $t_{a}^{(d)}$, compute the travel $\operatorname{cost} c_{k}^{r s(d)}$ of all valid routes, and then get the additional route flow $g_{k}^{r s(d)}$ through Eq.(1) and $f_{k}^{r s}=q_{r s} \cdot P^{r s}(k)$.

Step 5: Move. Let $f_{k}^{r s(d+1)}=f_{k}^{r s(d)}+\chi^{(d)}\left(g_{k}^{r s(d)}-f_{k}^{r s(d)}\right)$

where $\chi^{(d)}=\frac{1}{\beta(d)}$,

$\beta(d)=\left\{\begin{array}{l}\beta(d-1)+\lambda_{1}, \lambda_{1}>1, \text { if }\left\|\mathbf{g}^{(d)}-\mathbf{f}^{(d)}\right\| \geq\left\|\mathbf{g}^{(d-1)}-\mathbf{f}^{(d-1)}\right\| \\ \beta(d-1)+\lambda_{2}, 0<\lambda_{2}<1, \text { if }\left\|\mathbf{g}^{(d)}-\mathbf{f}^{(d)}\right\|<\left\|\mathbf{g}^{(d-1)}-\mathbf{f}^{(d-1)}\right\|\end{array}\right.$

Step 6: Test of convergence. The root mean squared error (RMSE) in Eq.(9) is adopted to test the convergence in this study.

$$
R M S E^{(d)}=\sqrt{\frac{1}{K} \sum_{r s} \sum_{k}\left(g_{k}^{r s(d)}-f_{k}^{r s(d)}\right)^{2}}
$$

where $K$ is the total number of valid routes between all OD pairs. The algorithm stops when $R M S E^{(d)}$ gets the specified convergence precision, otherwise let $d=d+1$, and return to Step 3 and continue calculate.

(2) Genetic algorithm design

Genetic algorithm includes the steps of coding, initial population generation, fitness function determined, selection, crossover and mutation, according to the characteristics of bi-level programming model of CNDP in this study, the genetic algorithm is designed as follows:
Step 1: Population initialization. Set various parameters of genetic algorithm, including the population size $N$, the maximum number of genetic generation $\bar{M}$, crossover probability $P_{c}$, mutation probability $P_{m}$. To improve the accuracy of this study, we use the real-coded method, and randomly generate $N$ chromosomes, let iteration number $\bar{b}=0$.

Step 2: Calculate fitness value. For each individual in the population, solve the PCL-SUE model in the lower level model according to the route-based self-regulated averaging algorithm shown in section 4.1 of this study, and obtain $N$ sets of equilibrium link flows $\left\{x_{a}\right\}$. Calculate the objective function values of the upper level model corresponding to the $N$ sets of capacity expansion on links, and then get fitness value of each individual.

Step 3: Selection, crossover and mutation. Selection operation uses the roulette selection, crossover operation uses the two points crossover and mutation operation uses uniform mutation. Through the above operations update chromosomes.

Step 4: Test of convergence. If the algorithm reaches the maximum number of genetic generation $\bar{M}$, the algorithm stops and outputs the best individual; if it does not reach the maximum number of genetic generation $\bar{M}$, then let $\bar{b}=\bar{b}+1$, return to Step 2 to continue the calculation.

\section{NUMERICAL EXAMPLE}

(1) Structure and parameters of numerical example

In order to verify the validity of the proposed algorithm, and compare with the existing traffic network design model, the common used example network in CNDP which is designed by Suwansirikul C et al. (1987) [15] is shown in Fig. (1). There are 4 nodes, 5 links and 3 valid paths in the traffic network. Path 1 is composed by link 1 and 4 , path 2 is composed by link 1, 3and 5, path 3 is composed by link 2 and 5. The length of each link is assumed equal and is $L$. This example is calculated by using the Matlab software on the computer with a $2.93 \mathrm{GHz}$ Intel Core I3 processor.

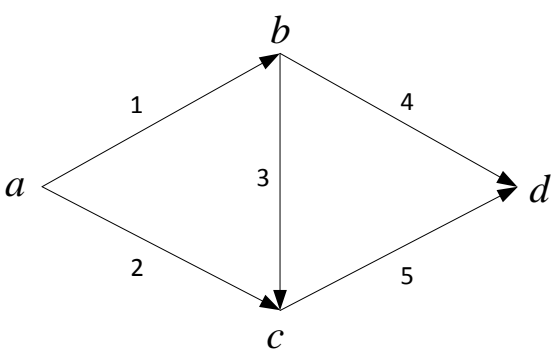

Fig. (1). Example traffic network.

Assume there is only 1 OD pair ad, total travel demand $q=100$, the BPR function added with $y_{a}$ is used as the travel cost function $t_{a}\left(x_{a}, y_{a}\right)$ of link $a$. 
Table 1. Freedom travel time, maximum capacities and parameter $h_{a}$ of different links.

\begin{tabular}{|c|c|c|c|c|c|}
\hline \multirow{2}{*}{ Parameters } & \multicolumn{5}{|c|}{ Link Number } \\
\cline { 2 - 6 } & $\mathbf{1}$ & $\mathbf{2}$ & $\mathbf{3}$ & $\mathbf{4}$ & 5 \\
\hline \hline$t_{a}(0) / \mathrm{min}$ & 4 & 6 & 2 & 5 \\
\hline$c_{a} /\left(\mathrm{pcu} \mathrm{i} \mathrm{h}^{-1}\right)$ & 40 & 40 & 60 & 40 \\
\hline$h_{a}$ & 2 & 2 & 1 & 2 \\
\hline
\end{tabular}

Table 2. Calculation result of network design with different $q$ •

\begin{tabular}{|c|c|c|c|c|c|c|c|c|c|}
\hline$q$ & $\begin{array}{c}\text { Lower Level } \\
\text { Model }\end{array}$ & $y_{1}$ & $y_{2}$ & $y_{3}$ & $y_{4}$ & $y_{5}$ & $D_{0}$ & $D$ & Improved Percentage \\
\hline \multirow{3}{*}{80} & UE & 0.48 & 0.45 & 0.03 & 0.34 & 0.4 & 824.59 & 822.39 & $0.27 \%$ \\
\hline & MNL-SUE & 0.64 & 0.29 & 0.04 & 0.20 & 0.56 & 844.41 & 841.75 & $0.32 \%$ \\
\hline & PCL-SUE & 0.57 & 0.34 & 0.00 & 0.25 & 0.47 & 831.64 & 829.29 & $0.28 \%$ \\
\hline \multirow{3}{*}{100} & UE & 1.34 & 1.21 & 0.01 & 0.97 & 1.10 & 1219.17 & 1200.59 & $1.52 \%$ \\
\hline & MNL-SUE & 1.52 & 0.99 & 0.01 & 0.73 & 1.29 & 1242.77 & 1223.86 & $1.52 \%$ \\
\hline & PCL-SUE & 1.43 & 1.12 & 0.02 & 0.87 & 1.18 & 1226.92 & 1208.28 & $1.52 \%$ \\
\hline \multirow{3}{*}{120} & UE & 3.26 & 2.55 & 0.29 & 2.11 & 2.39 & 1874.20 & 1777.98 & $5.13 \%$ \\
\hline & MNL-SUE & 3.00 & 2.26 & 0.12 & 1.77 & 2.55 & 1896.49 & 1800.30 & $5.07 \%$ \\
\hline & PCL-SUE & 2.87 & 2.41 & 0.03 & 1.95 & 2.44 & 1881.01 & 1784.31 & $5.14 \%$ \\
\hline \multirow{3}{*}{150} & UE & 6.06 & 5.50 & 0.02 & 4.64 & 5.28 & 3773.69 & 3156.24 & $16.36 \%$ \\
\hline & MNL-SUE & 6.26 & 5.24 & 0.09 & 4.28 & 5.46 & 3790.66 & 3176.98 & $16.19 \%$ \\
\hline & PCL-SUE & 6.15 & 5.39 & 0.23 & 4.57 & 5.36 & 3778.47 & 3162.25 & $16.31 \%$ \\
\hline
\end{tabular}

$t_{a}\left(x_{a}, y_{a}\right)=t_{a}^{0}\left[1+0.15\left(x_{a} /\left(c_{a}+y_{a}\right)\right)^{4}\right]$

where $t_{a}^{0}$ is free flow travel time of $\operatorname{link} a, x_{a}$ is flow on link $a, c_{a}$ is the maximum capacity of $\operatorname{link} a$.

Objective function:

$$
D=\sum_{a} t_{a}\left(x_{a}(\mathbf{y}), y_{a}\right) x_{a}(\mathbf{y})+1.5 \sum_{a} h_{a}\left(y_{a}\right)^{2} \text {. The free flow }
$$

travel time, maximum capacity and parameter $h_{a}$ of each link in paper [15] are shown in Table $\mathbf{1}$.

The other parameters used in the model are as follows: $\alpha_{m k}^{r s}, \mu_{m}^{r s}$ and $\sigma_{k j}^{r s}$ can be calculated directly from the network structure, dispersion coefficient $\theta=1$, parameters $\lambda_{1}=1.9$, $\lambda_{2}=0.1$, population size $N=50$, maximum number of genetic generation $\bar{M}=100$, crossover probability $P_{c}=0.40$, mutation probability $P_{m}=0.03$.
(2) Calculation results

The calculation results obtained by using the PCL-SUE model in the lower level model under different demand levels are shown in Table 2 . The total system costs $D_{0}$ (before the network design implement) and $D$ (after the network design implement) are shown in Table 2 . For ease of comparison, the calculation results of UE model and SUE model based on Logit model in the lower level model are also shown in Table 2.

As can be found from Table 2: (1) the results of network design are different when the lower level model of CNDP using different types of models to describe the behavior of users, which indicates that when the transportation planning managers adopt the network design model to assist the link expansion decisions, it needs to choose the model which could better describe the route choice behaviors of users according to the network characteristics, and ultimately to determine the final network design scheme through comparison; (2) through the comparison of the results of the UE 
Table 3. Calculation results of network design with different $\theta$.

\begin{tabular}{|c|c|c|c|c|c|c|c|c|c|}
\hline$\theta$ & $\begin{array}{c}\text { Lower Level } \\
\text { Model }\end{array}$ & $y_{1}$ & $y_{2}$ & $y_{3}$ & $y_{4}$ & $y_{5}$ & $D_{0}$ & $D$ & Improved Percentage \\
\hline \multirow{2}{*}{0.05} & MNL-SUE & 2.88 & 0.40 & 0.06 & 0.21 & 2.49 & 1406.69 & 1350.69 & $3.98 \%$ \\
\hline & PCL-SUE & 2.19 & 0.60 & 0.02 & 0.46 & 1.89 & 1294.14 & 1261.38 & $2.53 \%$ \\
\hline \multirow{2}{*}{0.1} & MNL-SUE & 2.50 & 0.45 & 0.06 & 0.35 & 2.26 & 1368.76 & 1325.95 & $3.13 \%$ \\
\hline & PCL-SUE & 1.98 & 0.70 & 0.01 & 0.49 & 1.75 & 1277.48 & 1249.95 & $2.16 \%$ \\
\hline \multirow{2}{*}{0.5} & MNL-SUE & 1.74 & 0.83 & 0.00 & 0.58 & 1.46 & 1269.44 & 1248.16 & $1.68 \%$ \\
\hline & PCL-SUE & 1.54 & 1.00 & 0.02 & 0.78 & 1.29 & 1236.75 & 1217.34 & $1.57 \%$ \\
\hline \multirow{2}{*}{1.0} & MNL-SUE & 1.52 & 0.99 & 0.01 & 0.73 & 1.29 & 1242.77 & 1223.86 & $1.52 \%$ \\
\hline & PCL-SUE & 1.43 & 1.12 & 0.02 & 0.87 & 1.18 & 1226.92 & 1208.28 & $1.52 \%$ \\
\hline \multirow{2}{*}{2.0} & MNL-SUE & 1.42 & 1.10 & 0.01 & 0.88 & 1.19 & 1228.61 & 1210.20 & $1.50 \%$ \\
\hline & PCL-SUE & 1.41 & 1.16 & 0.02 & 0.91 & 1.12 & 1222.06 & 1203.55 & $1.51 \%$ \\
\hline
\end{tabular}

model and other models, it can be found that the capacity expansion values of UE model on link 1 and 5 are smaller than the other models, however larger on link 2 and 4 . That is because the traffic flows assigned by UE model on link 1 and 5 are smaller than the other models, and larger on link 2 and 4 , which requires more investment to enhance the capacity on link 2 and 4, and so as to relieve congestion situation; (3) with the increase of the demand level $q_{a d}$, the congestion of the traffic network is also increased, thus it needs more investment to relieve traffic congestion, all of the links except link 3 need to increase investments. This also shows that during the network design process, the traffic planners should not only consider the current traffic demand level, but also consider the future growth in traffic demand, so as to make the traffic network design has certain forward-looking; (4) when it is in the same demand level and different traffic assignment models are used to describe the different behaviors of users, the improved proportion are nearly the same through the traffic network design, and with the increase of demand level $q_{a d}$, the percentage of improved in theory also increases, which indicates that when the road network has higher degree of congestion, under sufficient capital supply condition, the effect of traffic network design achieved is more obvious. However in fact, the government has a certain budget limit during the road expansion work, therefore the effect achieved by increasing road capacity is not necessarily very significant.

The results of traffic network design when the dispersion coefficient $\theta$ varies are shown in Table 3 .

As can be seen from Table 3: (1) With the increase of the dispersion coefficient $\theta$, the capacity expansion values of link 1 and 5 are decreased, and the capacity expansion values of link 2 and 4 are increased. That is because the flows assigned on link 1 and 5 are decreased and the flows on link 2 and 4 are increased when $\theta$ increases, thereby result in the corresponding changes occurs in the capacity expansion values. This shows that in the traffic network design in reality, it needs to survey the volumes and directions of traffic flows sufficiently, and get the reasonable value of dispersion coefficient $\theta$ through calibration based on the data obtained, otherwise it will lead to large errors in the results of assigned flows compared with actual situation because of inaccurate results, and then reduce the quality of network design scheme; (2) with the increasing of dispersion coefficient $\theta$, the improved percentage through the traffic network design is decreased, that is because the dispersion coefficient denotes the familiar degree of the traffic network. With the $\theta$ increases, the users could travel more reasonably, and the travel of users trend to be stabilized, therefore the improvement degree of the network through the network design reduces.

In order to compare the differences in the network design results of SUE and UE models when $\theta$ changes, the RMSE shown in Eq.(11) is adopted as the statistical indicator.

$$
R M S E=\sqrt{\frac{1}{|A|} \sum_{a}\left(y_{a}^{\mathrm{SUE}}-y_{a}^{\mathrm{UE}}\right)^{2}}
$$

where $|A|$ is the total number of links in the traffic network, $y_{a}^{\mathrm{UE}}$ denotes capacity expansion value of link $a$ obtained by UE model in the result of network design, $y_{a}^{\mathrm{SUE}}$ denotes capacity expansion value of link $a$ obtained by MNL-SUE or PCL-SUE model. The RMSE of different models under different $\theta$ are shown in Fig. (2).

As can be seen from Fig. (2): with the increase of the dispersion coefficient $\theta$, the RMSE indexes of both MNLSUE and PCL-SUE models used in the lower level model 


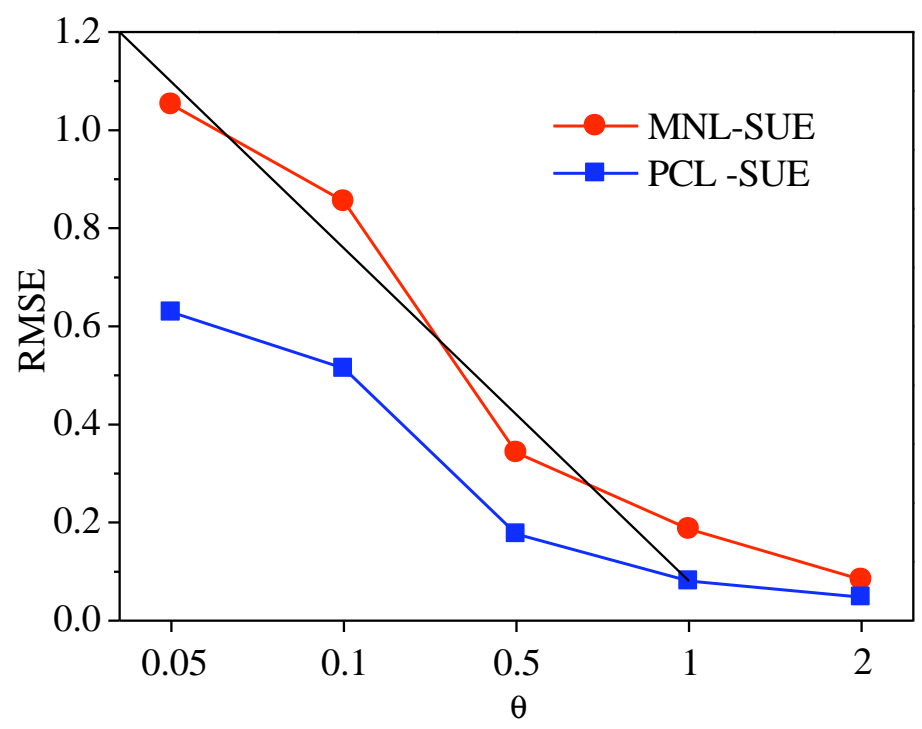

Fig. (2). RMSE under different $\theta$.

have downward trend, and when $\theta=2$, the RMSE indexes of different models are almost equal, that is because the increased $\theta$ implies that the users master more road network situations, the users' travel choices tend to be more closer to certainty choices, the assigned flows are also closer to the UE model, therefore the network design schemes by using the stochastic user equilibrium models in the lower level model are more closer to the results of UE model.

\section{CONCLUSION}

The stochastic user equilibrium model based on the paired combinatorial Logit model is adopted as the lower level model in this study and the continuous traffic network design model which could reflect the route choice behaviors of users objectively is designed. In order to improve the efficiency, for the lower level model, the route-based selfregulated averaging (SRA) algorithm is designed to solve the PCL-SUE model and for the upper level model, the genetic algorithm which has strong global search ability is adopted and the effectiveness of the proposed algorithm is verified through a numerical example. The calculation results of the proposed CNDP model with user equilibrium model, stochastic user equilibrium models based on Logit model and paired combinatorial Logit model in the lower level model are compared, and the impacts on the decision results of CNDP with different demand levels and dispersion coefficients are analyzed. In the future study, the network design model and algorithm which are proposed in this study could be applied to the actual traffic network design works, and the more efficient algorithms could be designed according to the characteristics of CNDP constructed in this study.

\section{CONFLICT OF INTEREST}

The authors confirm that this article content has no conflict of interest.

\section{ACKNOWLEDGEMENTS}

It is a project supported by the Major Program of the National Natural Science Foundation of China (Grant No. 71390332).

\section{REFERENCES}

[1] Z. Y. Gao, Y. F. Song, and B. F. Si, Urban Transportation Continuous Equilibrium Network Design Problem: Theory and Method, Beijing: Railway Publishing House of China, 2000.

[2] Y. F. Song, and Z. Y. Gao, "A heuristic solution algorithm for the continuous equilibrium network design problem," Journal of Beijing Jiaotong University, vol. 22, no. 6, pp. 19-24, 28, 1998.

[3] J. Yang, M. Xu, and Z. Y. Gao, "Sensitivity analysis of simulated annealing for continuous network design problems," Journal of Transportation Systems Engineering and Information Technology, vol. 9, no. 3, pp. 64-70, 2009.

[4] L. Xu, and Z. Y. Gao, "Urban transport network design based on link capacity reliability," China Journal of Highway and Transport, vol. 19, no. 2, pp. 86-90, 2006

[5] D. Z. W. Wang, and H. K. Lo, "Global optimum of the linearized network design problem with equilibrium flows," Transportation Research Part B, vol. 44, no. 4, pp. 482-492, 2010.

[6] S. -W. Chiou, "Bilevel programming for the continuous transport network design problem," Transportation Research Part B, vol. 39, no. 4, pp. 361-383, 2005.

[7] G. M. Wang, Z. Y. Gao, and M. Xu, "An MPEC formulation and its cutting constraint algorithm for continuous network design problem with multi-user classes," Applied Mathematical Modelling, vol. 38, no. 5-6, pp. 1846-1858, 2014.

[8] Z. Y. Gao, H. Z. Zhang, and H. J. Sun, "Bi-level programming models, approaches and applications in urban transportation network design problems," Journal of Transportation Systems Engineering and Information Technology, vol. 4, no. 1, pp. 35-44, 2004.

[9] H. Yang, and M. G. H. Bell, "Models and algorithms for road network design: a review and some new developments," Transport Reviews: A Transnational Transdisciplinary Journal, vol. 18, no. 3, pp. 257-278, 1998.

[10] K. E. Train, Discrete Choice Methods with Simulation, $2^{\text {nd }}$ Ed, United States of America: Cambridge University Press, 2003.

[11] D. McFadden, Modelling the Choice of Residential Location, United States of America: University of California, 1978. 
[12] J. N. Prashker, and S. Bekhor, "Stochastic user-equilibrium formulations for extended-logit assignment models," Transportation Research Record: Journal of the Transportation Research Board, vol. 1676, no. 1, pp. 145-152, 1999.

[13] M. Zhou, and S. D. Sun, Genetic Algorithms: Theory and Applications, Beijing: National Defence Industry Press, 1999.

[14] H. X. Liu, X. Z. He, and B. S. He, "Method of successive weighted averages (mswa) and self-regulated averaging schemes for solving stochastic user equilibrium problem," Networks and Spatial Economics, vol. 9, no. 4, pp. 485-503, 2009.

[15] C. Suwansirikul, T. L. Friesz, and R. L. Tobin, "Equilibrium decomposed optimization: a heuristic for the continuous equilibrium network design problem," Transportation Science, vol. 21, no. 4, pp. 254-263, 1987.

Received: November 20, 2014

(C) Li and Lang; Licensee Bentham Open.

This is an open access article licensed under the terms of the Creative Commons Attribution Non-Commercial License (http://creativecommons.org/licenses/by$\mathrm{nc} / 3.0 /$ ) which permits unrestricted, non-commercial use, distribution and reproduction in any medium, provided the work is properly cited. 